\title{
Time trend analysis of primary liver cancer incidence in Sihui county of Guangdong Province, China (1987-2011)
}

\author{
Wenyi Liu ${ }^{1,2+}$, Qing Liu ${ }^{1 \dagger}$, Qihong Huang ${ }^{3}$, Yuqiang $\mathrm{Lu}^{3}$, Shanghang Xie ${ }^{1}$, Aihua Lin ${ }^{2^{*}}$ and Sumei Cao ${ }^{1 *}$
}

\begin{abstract}
Background: Southern China is an endemic area for primary liver cancer (PLC), but it is unclear if rates have changed in recent decades. We evaluated PLC incidence and estimated the effects of age, period of diagnosis, and birth cohort in Sihui City, Guangdong Province, China.

Methods: Age-standardized rates (ASRs) of PLC were examined for both males and females from 1987 to 2011. Joinpoint regression analysis was conducted to estimate the annual percent changes in PLC incidence. The ageperiod-cohort (APC) model was used to investigate the effects of age, diagnosis period, and birth cohort on the relative risk (RR) of PLC.

Results: A total of 2988 PLC cases were identified in this period, with average ASRs of 51.1/100,000 for males and 11.7/100,000 for females. Joinpoint regression analysis revealed increasing PLC incidence throughout the entire period in both males (average annual change of $1.65 \%$ ) and females (0.20 \%). RRs increased gradually in both sexes from the youngest age group (30-34 years) to the oldest (80-84 years). In males, the RR decreased during diagnosis period from 1987-1991 to 1997-2001 and remained stable thereafter. In females, RRs fluctuated with diagnosis period throughout the entire period. Incidence tended to increase with birth cohort from 1905-1909 to 1975-1979 in both males and females; however, female incidence plateaued in the youngest cohorts born between 1955 and 1974, while incidence in males increased sharply in the cohorts born between 1965 and 1974. According to APC analysis, the full age-period-cohort (APC) model fit the data best, and the period-cohort (PC) model would be enough to explain variability of rates in females.
\end{abstract}

Conclusion: The PLC incidence rate in males of Sihui City has increased more significantly than female over the last 25 years. Despite the age effect in male, this trend mainly reflects the effects of risk factors that are present in early life (birth cohort) and period change in both genders.

Keywords: Primary liver cancer, Incidence trend, Joinpoint regression analysis, Age-period-cohort model

\section{Background}

Primary liver cancer (PLC) is one of the most common cancers worldwide, ranked the fifth most frequent malignancy in males (age-standardized rate (ASR) of 15.3/ 100,000 ) and the ninth in females (ASR of 5.4/100,000)

\footnotetext{
* Correspondence: linaihua@mail.sysu.edu.cn; caosm@sysucc.org.cn ${ }^{\dagger}$ Equal contributors

${ }^{2}$ School of Public Health, Sun Yat-sen University, 74 Zhongshan 2 road, Guangzhou, China

'Department of Cancer Prevention Research, Sun Yat-sen University Cancer Center, State Key Laboratory of Oncology in South China; Collaborative Innovation Center Medicine, 21 Qingcaigang, Jianshe 6 road, Guangzhou, Guangdong, China

Full list of author information is available at the end of the article
}

[1]. The geographic distribution of PLC is very uneven, with almost $85 \%$ of cases in developing countries and highest incidence rates in Southeast Asia and subSaharan Africa $[2,3]$. In most populations, the major histological type of PLC is hepatocellular carcinoma (HCC), followed by cholangiocarcinoma (CC) and combined hepatocellular and cholangiocarcinoma (cHCC-CC) [4].

Up to $80 \%$ of PLC worldwide is associated with chronic hepatitis $B$ virus (HBV) and hepatitis $C$ virus (HCV) infections [5]. Infection by HBV is the most common risk factor for PLC in Asia, and prospective cohort 
studies have found a 5- to 100-fold increase in HCC risk among persons chronically infected with $\operatorname{HBV}[5,6]$. Exposure to aflatoxin, a mycotoxin from Aspergillus molds, is the second most common cause in most endemic regions of China [7]. In southern China, including Guangdong and Guangxi Provinces, liver and biliary disorders, especially cholangiocarcinoma, are primarily associated with infection by the liver fluke Clonorchis sinensis $[8,9]$. Other possible environmental and lifestyle risk factors for PLC have also been identified, such as alcohol-related liver diseases, obesity, and smoking $[10,11]$.

In China, PLC was the third most common cancer in males $(268,757$ new cases with ASR of $32.2 / 100,000)$ and the fifth most common in females $(90,083$ new cases with ASR of 10.4/100,000) in 2010 [12]. According to the first national death study, the endemic are located in the southern, southeastern, southwestern and northeastern areas of China, such as Guangdong, Jiangsu, Zhejiang, Guangxi and Heilongjiang [13]. Several studies have reported a decreasing trend in some epidemic regions of China, such as Shanghai, Qidong and Fusui [14-16], but changes in PLC incidence have not been examined in southern China. The objective of our study was to describe the changes in PLC incidence during the last 25-year period (1987-2011) in Sihui, Guangdong Province, southern China. These changes may identify modifiable environmental and lifestyle factors for PLC prevention and control.

\section{Methods}

\section{Study setting}

Sihui is a county-level city located in the central part of Guangdong Province. In 2011, the population of Sihui was 418,097 and $>75 \%$ lived in rural residences. The major industries include jade processing, smelting, metallurgic casting, and ceramic manufacturing. Climate in Sihui is warm and humid all year around, and the main economic crops are rice and oranges. According to previous reports, the most common malignancy in Sihui is PLC, accounting for $24.56 \%$ of the total [17, 18]. Thus, PLC is an enormous economic and social burden in this region.

\section{Data sources}

Since the establishment of a national three-level cancer network in 1987, a malignant tumor registration report system has been implemented in Sihui [19]. All cancer cases are regularly reported (approximately once a month) by local general practitioners to the central hospital of each town, and then reported to Sihui Cancer Institute once every 3 months. Health specialists assigned by Sihui Cancer Institute collect the reports, record the information onto predesigned cards, and check the quality of the data. To reduce the possibility of missing reports, cancer institute staff also collect the tumor data from hospitals in Sihui once every half year since 1998. For this study, the demographic data (age composition by sex) from 1987 to 2011 were collected from the population information published by the Sihui Statistics Department.

During the 1987-2011 period, 2988 cases of malignant neoplasm of the liver $(\mathrm{ICD} 10=\mathrm{C} 22)$ were identified from Sihui Cancer Registry Institute. The basic registration data, including sex, age, birth date, family history, pathological basis, diagnostic date, disease stage at diagnosis, International Classification of Diseases-10th revision (ICD-10) code, cause of death, registered identification number, and national identification number, were collected from the cancer reporting cards. Among 2988 cases, 675 cases (22.59\%) were from the histologic diagnosis, 79 cases (2.64\%) were from cytological or biochemical or immune deduction, 1674 cases (56.02 \%) were from BUltrasonograph or other medical imaging, and 373 cases $(12.49 \%)$ were from clinical detection. The number of death certification notifications (DCN) cases was $187(6.26 \%)$, and the ratio of mortality to incidence (M: I) was $94.88 \%$. This study was approved by the Institutional Research Ethics Committee of Sun Yat-sen University Cancer Center (YB2016-034). In this study, only annual cancer registration report data was used and no information to identify individual subjects was included.

\section{Statistical methods}

In order to estimate age-standardized incidence rates of liver cancer, data stratified by sex were arranged into five shorter intervals (1987-1991, 1992-1996, 1997-2001, 2002-2006 and 2007-2011) for the time period 1987-2011. All age-adjusted incidence rates were calculated using direct standardization with the Segi's World Standard Population (1960) [20].

Joinpoint regression was used to estimate annual percent changes (EAPCs). Significant joinpoints were identified by permutation tests using $95 \%$ of the asymptote [21], starting with no joinpoints and adding up to three to the model [22].

An age-period-cohort (APC) model was used to estimate the incidence of PLC. Regression analysis considered the effects of three factors on incidence for each gender: age, period, and cohort [23]. The model assumed that the number of liver cancer cases follows a Poisson distribution and that incidence rates are a multiplicative function of the included model parameters, making the logarithm of the rates an additive function of the parameters [24, 25]. Relative risks 
(RRs) were calculated to show the effects of age, diagnosis period, and birth cohort in the APC models. All analyses were restricted to the 30-84 years age range and data were categorized into 11 age groups from 30-34 years to 80-84 years when applied to the APC model, together with 5 diagnosis period groups (1987-1991 to 2007-2011) and 15 birth cohort groups (1905-1909 to 1975-1979) (Table 4 in Appendix). To make this model more stable, we chose the 55-59 year age group, the 1997-2001 diagnosis period group, and the 1940-1944 birth cohort group as references instead of the first or the last group [26]. Goodness of fit of the models was assessed both by deviance and Akaike's information criterion (AIC). Ratios of deviance to the degrees of freedom were closer to 1 and lower AIC values indicated a better fit [27, 24, 25]. All analyses were performed using SAS 9.3 (Version 9.3, SAS Institute Inc., NC, USA).

\section{Results}

\section{PLC incidence in Sihui}

Over the 25-year study period from 1987 to 2011, 2988 PLC cases were identified in Sihui. Of these, 2380 (79.7 \%) were diagnosed in men and 608 (20.3\%) in women. The crude incidences were 33.74/ 100,000 for the total population, 47.01/100,000 for males, and 12.64/100,000 for females between 1987 and 2011. The corresponding ASRs were 30.83/ 100,000 for the total population, 51.14/100,000 for males, and 11.69/ 100,000 for females. The overall age-adjusted rates among males and females increased in this period, from $32.49 / 100.000$ to $69.54 / 100,000$ in male and $7.03 / 100,000$ to $13.43 / 100,000$ in female (Table 1, Fig. 1).

\section{Joinpoint regression analysis of PLC incidence}

During the 25-year period, three-joinpoint model was obtained as the best model for both sexes, and the incidence of PLC fluctuated with increasing trend at an average annual percent change of $1.65 \%$ for males and $0.20 \%$ for females (Fig. 2).

\section{Age-period-cohort effects on PLC incidence}

In males, the full APC model fit the data best with the Deviance $=42.96$, AIC $=10352$. Among females, a period-cohort model was sufficient to explain variability of the PLC incidence, since a non-significant ( $\triangle$ Deviance) between the age-period-cohort (Deviance $=28.95)$ and the period-cohort (Deviance $=42.19 ; p=$ $0.152)$ models was found. The $\Delta$ deviance of corresponding models of age-period $(\Delta$ deviance $=47.04)$ was higher than period-cohort $(\Delta$ deviance $=13.24)$ indicated that the birth cohort effect was more important than the age effect in female (Table 2). The component effects in the final APC model are shown in Table 3. The age effects for both sexes showed that the risk of PLC had consistent increasing trends with age. Compared with the age group of 55-59 years, the RR of PLC was up to 3.61(95\% CI:2.20-5.96) for males and 3.86 (95\% CI:1.78-8.36) for females in the oldest age group (80-84 years) (Fig. 3a). Period effects for males depicted that the RRs decreased from 1987-1991 to 1997-2001, and then plateaued afterwards; for females, RRs fluctuated through the entire period (Fig. 3b). By using the 1940-1944 birth cohort as the reference group, an obvious increased incidence trend in both males and females were obtained; however, females had a plateau in the youngest cohorts who were born during 1955-1974 and males had a sharp increasing trend in the cohorts who were born during 1965-1974 (Fig. 3c).

\section{Discussion}

This study reveals that the incidence rate of PLC in Sihui $(30.8 / 100,000)$ was substantially higher than the national level $(10.1 / 100,000)$ during the period 19872011 [1]. Moreover, the average ASR in this region exhibited an increasing trend over this period, in contrast to three other endemic regions of China, Shanghai [14], Qidong [15], and Fusui [16], where incidence rates are stable or declining. The APC analysis revealed that the increasing trend of PLC incidence in Sihui is primarily due to the effects of period changes and a spike in the younger cohorts, particularly males born between 1965 and 1974. Such a pattern may indicate the impact of lifestyle and environmental changes on the risk of PLC in rural areas of southern China.

Reasons for the high incidence of PLC in Sihui are not completely clear but may be related to higher HBV infection rates and other possible infection risk factors compared to other regions of China. A survey conducted between 1992 and 1995 reported the highest HBV infection rate in Guangdong compared to the rest of China, with $\mathrm{HBs}-\mathrm{Ag}$ positive rate of $17.85 \%$ in the general population and $19.86 \%$ in children aged $<15$ years. Among children aged $<15$, age-specific prevalence rates of $\mathrm{HBs}-\mathrm{Ag}$ was 23.68, $14.63,17.5319 .6118 .71$, and $23.84 \%$ in the 6 age groups (1-, 2-3, 4-5, 6-7, 8-9, 10-14 years), respectively [28-30]. Hence, reducing HBV infection is critical for controlling PLC in southern China. Hepatitis $B$ vaccination of neonates and infants is the most effective way of eliciting protective antibodies to HBV surface antigen (anti-HBs) and in reducing the prevalence of HBs-Ag among children [31, 32]. Since 2002, subsidy for HBV vaccination has been 
Table 1 Age-standardized rates of primary liver cancer incidence in Sihui City, Guangdong Province, China from 1987 to 2011

\begin{tabular}{|c|c|c|c|c|c|c|}
\hline \multirow[t]{2}{*}{ Year } & \multicolumn{3}{|l|}{ Males } & \multicolumn{3}{|c|}{ Females } \\
\hline & Cases & Crude incidence $(/ 100,000)$ & $\operatorname{ASR}(/ 100,000)$ & Cases & Crude incidence $(/ 100,000)$ & $\operatorname{ASR}(/ 100,000)$ \\
\hline 1987 & 50 & 29.27 & 32.49 & 13 & 7.66 & 7.03 \\
\hline 1988 & 61 & 35.30 & 39.35 & 12 & 7.06 & 6.56 \\
\hline 1989 & 68 & 39.32 & 43.50 & 17 & 9.79 & 8.93 \\
\hline 1990 & 80 & 44.69 & 48.72 & 19 & 10.87 & 11.14 \\
\hline 1991 & 82 & 46.07 & 49.74 & 23 & 13.03 & 11.38 \\
\hline 1992 & 96 & 52.70 & 55.36 & 31 & 17.46 & 15.84 \\
\hline 1993 & 102 & 55.06 & 58.87 & 24 & 13.40 & 13.09 \\
\hline 1994 & 72 & 38.19 & 40.19 & 24 & 13.31 & 12.45 \\
\hline 1995 & 97 & 50.88 & 52.39 & 35 & 19.22 & 17.76 \\
\hline 1996 & 70 & 36.17 & 41.28 & 23 & 12.53 & 13.62 \\
\hline 1997 & 81 & 41.29 & 49.69 & 29 & 15.66 & 15.18 \\
\hline 1998 & 68 & 33.85 & 38.25 & 22 & 11.67 & 11.12 \\
\hline 1999 & 78 & 38.69 & 44.73 & 20 & 10.50 & 10.16 \\
\hline 2000 & 93 & 45.92 & 48.15 & 31 & 16.22 & 14.01 \\
\hline 2001 & 76 & 37.29 & 38.42 & 22 & 11.44 & 10.72 \\
\hline 2002 & 84 & 41.09 & 42.85 & 20 & 10.34 & 9.86 \\
\hline 2003 & 92 & 44.72 & 43.94 & 21 & 10.82 & 9.22 \\
\hline 2004 & 121 & 58.29 & 62.15 & 15 & 7.73 & 6.40 \\
\hline 2005 & 117 & 55.57 & 56.88 & 26 & 13.31 & 10.30 \\
\hline 2006 & 114 & 53.82 & 55.28 & 21 & 10.63 & 9.54 \\
\hline 2007 & 140 & 66.11 & 67.72 & 29 & 14.67 & 12.04 \\
\hline 2008 & 140 & 66.38 & 69.36 & 33 & 16.50 & 14.52 \\
\hline 2009 & 126 & 59.62 & 62.12 & 39 & 19.31 & 16.08 \\
\hline 2010 & 133 & 62.76 & 66.30 & 25 & 12.30 & 10.28 \\
\hline 2011 & 139 & 65.08 & 69.54 & 34 & 16.62 & 13.43 \\
\hline total & 2380 & 47.01 & 51.14 & 608 & 12.64 & 11.69 \\
\hline
\end{tabular}

ASR Age-standardized rates

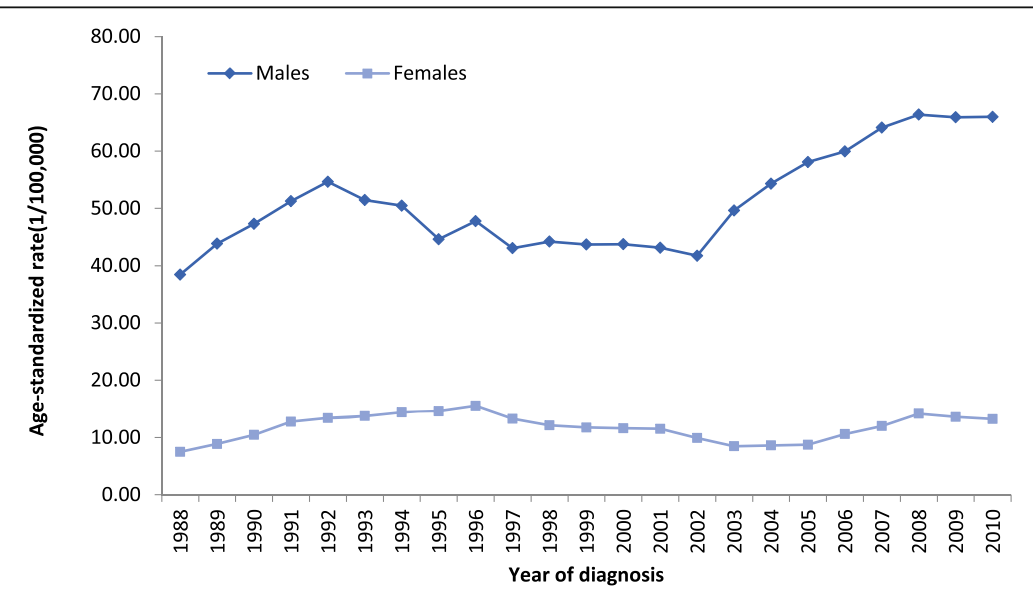

Fig. 1 Age-standardized rates of primary liver cancer incidence by sex using the moving average in three-year intervals in Sihui, China, 1987-2011. The overall age-adjusted rate among males and females was increased in this period, from 32.49/100.000 to 69.54/100,000 in male and 7.03/100,000 to 13.43/ 100,000 in female (Fig. 1) 

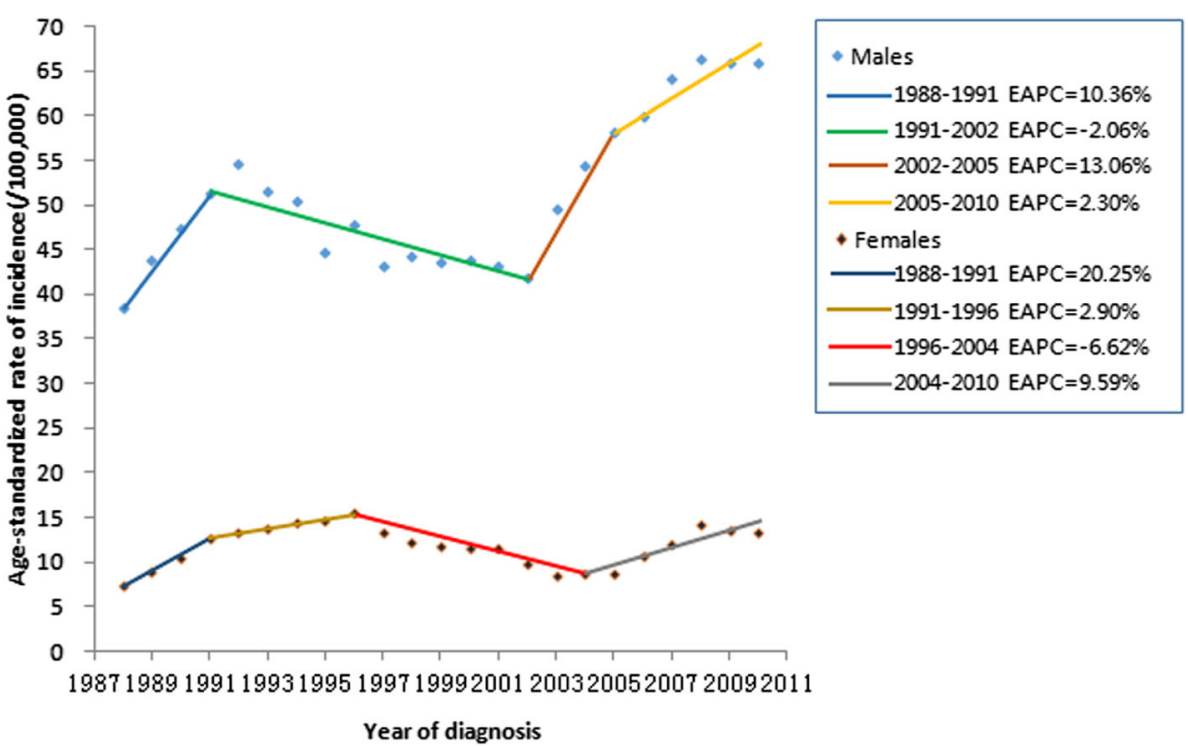

Fig. 2 Trends in world age-standardized rates (ASR-W) of PLC incidence for both sexes in the period of 1987-2010 in Sihui, China, with joinpoint regression. During the 25-year period, three-joinpoint model were obtained as the best model for both sexes and the incidence of PLC fluctuated with increasing trend at an average annual percent change of $1.65 \%$ for males and $0.20 \%$ for females (Fig. 2)

provided for all infants and has thereafter largely reduced HBV infection rate among Chinese children $[31,32]$. However, HBV infection remains highly prevalent in the population born before the vaccination program and the incidence of PLC has remained high in adults. Since hepatitis B vaccine is proven to prevent HBV infection among children and teenagers, it may also prove beneficial to young adults in preventing HBV induced PLC [31].
Clonorchis sinensis (C. sinensis) infection is another possible contributor to the high incidence of PLC in Sihui. Clonorchiosis is also common in Guangdong, with heavily endemic areas located along the Pearl River Delta [33]. Raw freshwater fish is a staple of the Cantonese diet in this region, and these fish act as the second intermediate host in the lifecycle of $C$. sinensis. Research showed that Clonorchis sinensis infection is strongly related to liver and biliary disorders, especially

Table 2 Age-period-cohort models for PLC incidence rates in Sihui, China

\begin{tabular}{|c|c|c|c|c|c|c|c|}
\hline & DF & Deviance & Deviance/DF & $\Delta$ Deviance & $\triangle \mathrm{DF}$ & $P$ & $\mathrm{AlC}$ \\
\hline \multicolumn{8}{|l|}{ Male } \\
\hline Age & 44 & 157.34 & 3.58 & 114.38 & 17 & $<0.001$ & 10432 \\
\hline Period & 50 & 1103.36 & 22.07 & 1060.04 & 23 & $<0.001$ & 11366 \\
\hline Cohort & 40 & 507.06 & 12.68 & 464.04 & 13 & $<0.001$ & 10790 \\
\hline Age-period & 40 & 87.76 & 2.19 & 44.8 & 13 & $<0.001$ & 10370 \\
\hline Age-cohort & 30 & 69.51 & 2.31 & 26.55 & 3 & $<0.001$ & 10372 \\
\hline Period-cohort & 36 & 140.28 & 3.9 & 97.32 & 9 & $<0.001$ & 10431 \\
\hline Age-period-cohort & 27 & 42.96 & 1.59 & Reference & Reference & Reference & 10352 \\
\hline \multicolumn{8}{|l|}{ Female } \\
\hline Age & 44 & 98.48 & 2.24 & 69.53 & 17 & $<0.001$ & 11483 \\
\hline Period & 50 & 407.97 & 8.16 & 379.02 & 23 & $<0.001$ & 11780 \\
\hline Cohort & 40 & 113.21 & 2.83 & 84.26 & 13 & $<0.001$ & 11505 \\
\hline Age-period & 40 & 75.99 & 1.9 & 47.04 & 13 & $<0.001$ & 11468 \\
\hline Age-cohort & 30 & 47.2 & 1.57 & 18.25 & 3 & $<0.001$ & 11459 \\
\hline Period-cohort & 36 & 42.19 & 1.17 & 13.24 & 9 & 0.152 & 11443 \\
\hline Age-period-cohort & 27 & 28.95 & 1.07 & Reference & Reference & Reference & 11447 \\
\hline
\end{tabular}

DF Degree of freedom, $\triangle$ Deviance increase in deviance from the APC model, $\triangle D F$ increase in DF from the APC model, AIC Akaike's information criterion 
Table 3 Relative risk (RR) and $95 \%$ confidence interval (Cl) of PLC incidence for both sexes by age-period-cohort model in Sihui, China

\begin{tabular}{|c|c|c|c|c|c|}
\hline \multirow[t]{2}{*}{ Parameter } & \multirow[t]{2}{*}{ Subgroup } & \multicolumn{2}{|c|}{ Males } & \multicolumn{2}{|c|}{ Females } \\
\hline & & $\mathrm{RR}$ & $95 \% \mathrm{Cl}$ & $\mathrm{RR}$ & $95 \% \mathrm{Cl}$ \\
\hline \multirow[t]{11}{*}{ Age (years) } & $30-34$ & 0.04 & $0.03-0.07$ & 0.12 & $0.05-0.28$ \\
\hline & $35-39$ & 0.09 & $0.07-0.13$ & 0.22 & $0.11-0.44$ \\
\hline & $40-44$ & 0.23 & $0.18-0.30$ & 0.42 & $0.24-0.72$ \\
\hline & $45-49$ & 0.39 & $0.31-0.48$ & 0.45 & $0.28-0.73$ \\
\hline & $50-54$ & 0.63 & $0.53-0.75$ & 0.87 & $0.59-1.28$ \\
\hline & $55-59$ & \multicolumn{2}{|c|}{ Reference } & \multicolumn{2}{|c|}{ Reference } \\
\hline & $60-64$ & 1.24 & $1.03-1.49$ & 1.54 & $1.06-2.21$ \\
\hline & $65-69$ & 1.96 & $1.57-2.45$ & 2.27 & $1.48-3.49$ \\
\hline & $70-74$ & 2.36 & $1.78-3.12$ & 2.49 & $1.48-4.18$ \\
\hline & $75-79$ & 3.23 & $2.26-4.61$ & 2.80 & $1.49-5.26$ \\
\hline & $80-84$ & 3.61 & $2.20-5.96$ & 3.86 & $1.78-8.36$ \\
\hline \multirow[t]{5}{*}{ Period } & 1987-1991 & 1.70 & $1.33-2.18$ & 1.05 & $0.66-1.67$ \\
\hline & 1992-1996 & 1.50 & $1.25-1.80$ & 1.35 & $0.97-1.89$ \\
\hline & 1997-2001 & \multicolumn{2}{|c|}{ Reference } & \multicolumn{2}{|c|}{ Reference } \\
\hline & $2002-2006$ & 0.96 & $0.86-1.07$ & 0.70 & $0.55-0.88$ \\
\hline & $2007-2011$ & 1.00 & $0.91-1.25$ & 1.00 & $0.61-1.52$ \\
\hline \multirow[t]{13}{*}{ Cohort } & 1910-1914 & 0.11 & $0.04-0.29$ & 0.17 & $0.04-0.68$ \\
\hline & 1915-1919 & 0.15 & $0.08-0.34$ & 0.08 & $0.02-0.34$ \\
\hline & 1920-1924 & 0.20 & $0.12-0.32$ & 0.44 & $0.20-0.96$ \\
\hline & 1925-1929 & 0.46 & $0.32-0.65$ & 0.49 & $0.26-0.93$ \\
\hline & 1930-1934 & 0.52 & $0.39-0.69$ & 1.02 & $0.62-1.67$ \\
\hline & 1935-1939 & 0.80 & $0.64-1.00$ & 1.00 & $0.65-1.52$ \\
\hline & 1940-1944 & 0.88 & $0.73-1.06$ & 1.09 & $0.75-1.58$ \\
\hline & 1945-1949 & \multicolumn{2}{|c|}{ Reference } & \multicolumn{2}{|c|}{ Reference } \\
\hline & 1950-1954 & 1.51 & $1.24-1.82$ & 1.67 & $1.12-2.50$ \\
\hline & 1955-1959 & 2.03 & $1.63-2.54$ & 1.68 & $1.04-2.70$ \\
\hline & 1960-1964 & 2.22 & $1.70-2.90$ & 1.24 & $0.69-2.23$ \\
\hline & 1965-1969 & 2.60 & $1.88-3.61$ & 1.59 & $0.78-3.21$ \\
\hline & 1970-1974 & 4.18 & $2.84-6.15$ & 1.13 & $0.47-2.71$ \\
\hline
\end{tabular}

cholangiocarcinoma [9]. However, we were unable to differentiate between types of PLC because the proportion of histologically diagnosed PLC was small (9.14 \%) and only $2.5 \%$ of the histologically diagnosed PLC cases can be classified as a specific pathological subtype. Given the symptoms of PLC are insidious at onset, progress quickly and with high mortality, the disease is usually diagnosed when the tumor is large and incurable. Therefore, patients often refuse the pathological biopsy diagnosis and treatment. Increasing the early detection rate and treatment effect might improve the pathologic diagnostic rate of PLC in the future. Another limitation is we cannot analyze the effect of risk factors clearly for PLC cases due to the lack of environmental risk factors exposure information.
The APC analysis suggests that population aging is one of the effectors of the increasing PLC incidence in both sexes. Indeed, PLC incidence increased progressively with age, peaking in the $80-84$ year age group. With economic development and introduction of modern medical technology, the proportion of elderly in the general population has increased dramatically. In 2010, the average life expectancy in Sihui (76.6 years) was longer than the national by 3.1 years [34]. Age increases PLC risk due to the long incubation process (about 50 years) from HBV infection to PLC [35, 36], possibly conferring higher PLC risk for the given infection rate.

Variations in incidence over long periods often reflect the impacts of new diagnostic techniques, improved medical interventions, and greater accuracy in determining the causes of morbidity and mortality. According to the APC model, PLC incidence rates were highest for both sexes in the first diagnostic period included (1987-1991). In females, this period was followed by a general decreasing trend, while in males the rate trended downward only until 19972001 and has since remained stable. The peak detection of PLC in the 1987-1991 period may be due to the widespread introduction of ultrasound and AFP measurement [10]. In the 1980s, ultrasound examination together with AFP testing has been gradually popularized as a PLC screening method in the endemic areas in China, which led to a large number of asymptomatic cases of PLC found and more frequent neoplasms identified from cirrhotic patients [37, 38].

A birth cohort effect usually reflects early life exposure to specific risk factors that were absent in other periods [39]. In our study, birth cohortstratified incidences in males and females showed two trends. Before the 1947-1951 cohort, a slow progressive increase in PLC incidence was observed in both males and females. However, there was a sharp acceleration from the 1952-1956 to the 19681972 cohort in younger males, whereas the equivalent female cohorts showed only a narrow fluctuation. We suggest that the combination of high HBV and $C$. sinensis infection rates, exposure to cigarette smoking and alcohol drinking in the younger male generation may be the main reasons underlying this birth cohort effect in males of Sihui. It has been reported that males had higher prevalence rate than females for HBs-Ag (23.56 vs $15.55 \%, p<0.05)$ [29] and for $C$ sinesis $(18.92$ vs $13.89 \%, p<0.05)$ [33]. Several studies conducted in 1980s-1990s reported that the prevalence rates of cigarette smoking were 15 times higher (39.04 vs.2.47 \%) and alcohol drinking 21 times higher (38.24 vs $1.77 \%$ ) in the male younger cohorts than females [40,41]. These differences might result in a more rapid increase in PLC 
A Age effects

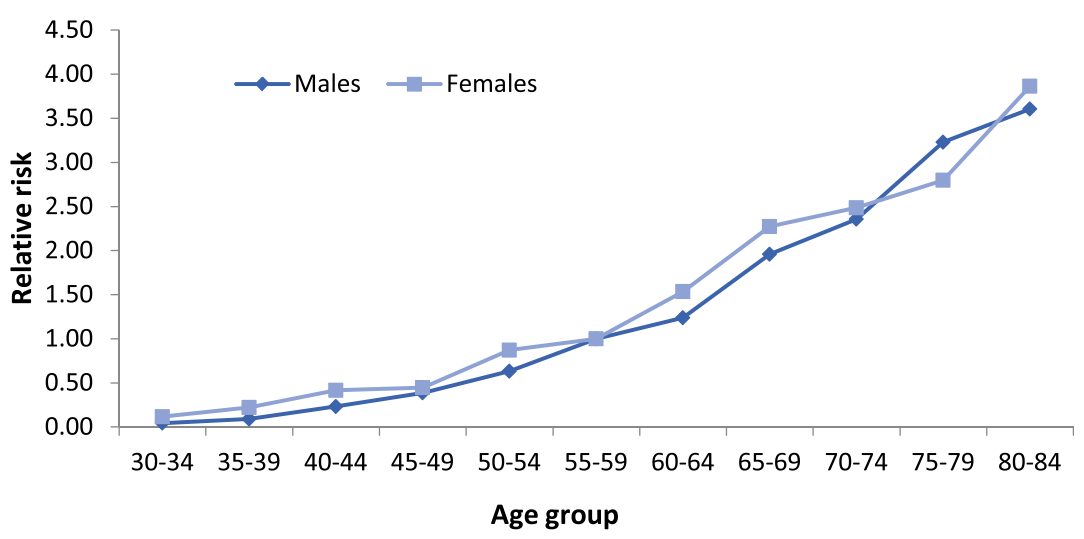

B Period effects

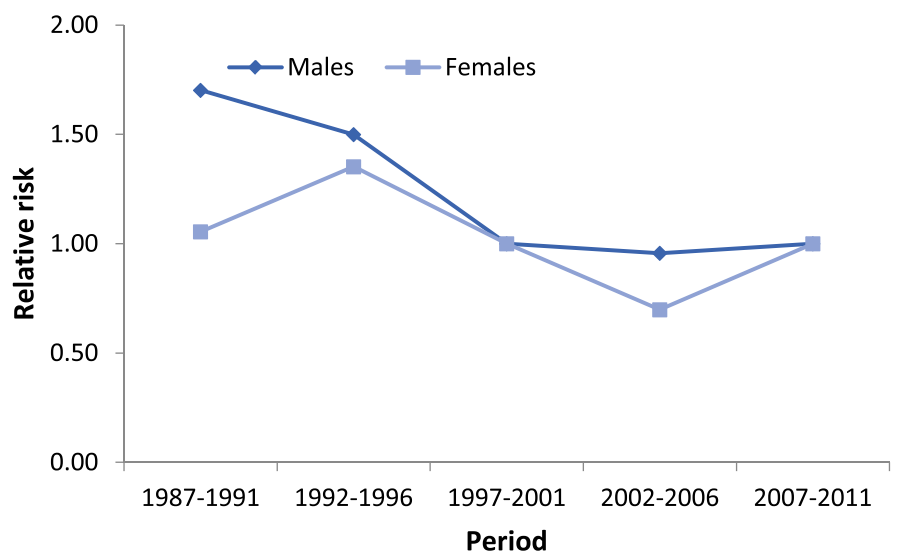

C Birth cohort effects

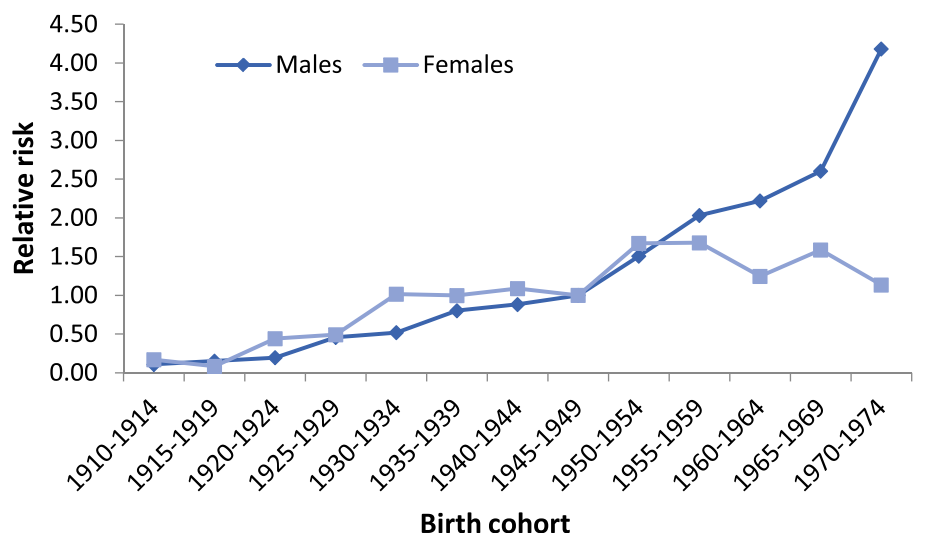

Fig. 3 Effect of age, period, and birth cohort on the relative risk of primary liver cancer incidence with APC regression analysis in Sihui county by sex. a Age effects; $\mathbf{b}$, Period effects; $\mathbf{c}$, Birth cohort effects. The age effects for both sexes showed that the risk of PLC increased with age. Compared with the age group of 55-59 years, the RR of PLC was up to 3.61(95 \% Cl:2.20-5.96) for males and 3,86 (95\% Cl:1.78-8.36) for females in the oldest age group (80-84 years) (Fig. 3a). Period effects for males depicted that the RRs decreased from 1987-1991 to 1997-2001, and then plateaued afterwards; for females, RR fluctuated through the entire period. The RRs increased from period1987-1991 to 1992-1996, decreased consistently from 1992-1996 to 2002-2006, and then increased to the staring risk (Fig. 3b). By using the 1940-1944 birth cohort as the reference group, an obvious increased incidence trend in both males and females were obtained; however, females had a plateau in the youngest cohorts who were born during 1955-1974 and males had a sharp increasing trend in the cohorts who were born during 1965-1974 (Fig. 3c) 
incidence in men than women. More detailed infor-

Table 4 Incidence rates of PLC in different age groups, period groups and birth cohort groups for both sexes in Sihui, China

\begin{tabular}{|c|c|c|c|c|c|}
\hline \multirow[t]{2}{*}{ Variable } & \multirow[t]{2}{*}{ Subgroup } & \multicolumn{2}{|l|}{ Males } & \multicolumn{2}{|c|}{ Female } \\
\hline & & Cases & Incidence rate $(/ 100,000)$ & Cases & Incidence rate $(/ 100,000)$ \\
\hline \multirow[t]{11}{*}{ Age (Years) } & $30-34$ & 95 & 21.87 & 15 & 3.67 \\
\hline & $35-39$ & 166 & 41.62 & 29 & 7.80 \\
\hline & $40-44$ & 252 & 85.88 & 45 & 16.60 \\
\hline & $45-49$ & 279 & 106.90 & 42 & 17.63 \\
\hline & $50-54$ & 303 & 140.92 & 62 & 31.02 \\
\hline & $55-59$ & 311 & 180.80 & 60 & 34.45 \\
\hline & $60-64$ & 279 & 169.30 & 81 & 49.21 \\
\hline & $65-69$ & 267 & 208.64 & 90 & 57.91 \\
\hline & $70-74$ & 181 & 194.75 & 71 & 53.83 \\
\hline & $75-79$ & 116 & 191.44 & 45 & 44.89 \\
\hline & $80-84$ & 44 & 139.91 & 31 & 40.90 \\
\hline \multirow[t]{5}{*}{ Period } & 1987-1991 & 323 & 34.02 & 78 & 8.43 \\
\hline & 1992-1996 & 422 & 41.82 & 129 & 13.49 \\
\hline & 1997-2001 & 379 & 37.71 & 379 & 40.00 \\
\hline & $2002-2006$ & 506 & 48.65 & 96 & 9.85 \\
\hline & 2007-2011 & 659 & 62.20 & 152 & 15.09 \\
\hline \multirow[t]{13}{*}{ Cohort } & 1910-1914 & 19 & 103.88 & 3 & 8.89 \\
\hline & 1915-1919 & 36 & 105.69 & 22 & 39.58 \\
\hline & 1920-1924 & 116 & 195.60 & 32 & 37.57 \\
\hline & 1925-1929 & 155 & 172.57 & 85 & 67.98 \\
\hline & 1930-1934 & 253 & 207.60 & 79 & 51.63 \\
\hline & 1935-1939 & 268 & 167.97 & 77 & 58.00 \\
\hline & 1940-1944 & 236 & 138.97 & 54 & 32.52 \\
\hline & 1945-1949 & 274 & 137.20 & 65 & 36.82 \\
\hline & 1950-1954 & 207 & 79.51 & 57 & 24.06 \\
\hline & 1955-1959 & 232 & 74.73 & 37 & 12.62 \\
\hline & 1960-1964 & 167 & 60.89 & 31 & 11.87 \\
\hline & 1965-1969 & 141 & 58.27 & 15 & 6.56 \\
\hline & 1970-1974 & 74 & 37.26 & 7 & 3.79 \\
\hline
\end{tabular}

mation on the risk factors for PLC should be gathered from southern China.

\section{Conclusions}

A significant increase in the incidence rate of primary liver cancer was observed in Sihui from 1987 to 2011 for both sexes. Estimates from cohort effect showed that the increasing trend in male is likely to continue over the next decade in male, while female tended to leveled off since 1950. Although the reasons for increasing PLC incidence in Sihui are not fully clear, higher prevalences of HBV and C. sinensis infection are likely contributors.
Further research exploring the prevalence of possible risk factors associated with PLC is needed to formulate a comprehensive PLC prevention and control strategy in this region of southern China.

\section{Appendix}

\section{Abbreviations}

AFP: a-fetoprotein; AIC: Akaike's information criterion; anti-HBs: Antibodies to HBV surface antigen; APC: Age-Period-Cohort model; ASRs: Age-standardized rates; C. sinensis: Clonorchis sinensis; CC: Cholangiocarcinoma; CHCC-CC: Combined Hepatocellular and cholangiocarcinoma; DCN: Death Certification Notifications; DF: Degree of freedom; EAPC: Estimated annual percent change; EAPCs: Estimate annual percent changes; HBs-Ag: HBV surface antigens; HBV: Hepatitis B virus; 
HCC: Hepatocellular carcinoma; HCV: Hepatitis C virus; ICD-10: International Classification of Diseases-10th revision; M:l: The Ratio of Mortality to Incidence: PLC: Primary Liver Cancer; RR: Relative Risk; $\triangle$ Deviance: Increase in deviance from the APC model; $\triangle \mathrm{DF}$ : Increase in DF from the APC model

\section{Acknowledgment}

We appreciate all staff of the Sihui Cancer Registry for their work on registration and follow-up.

\section{Funding}

This study was supported by a grant from the National Natural Science Foundation of China (81373068).

\section{Availability of data and materials}

We are unable to share the data owing to the grounds of the ethical approval. Most of the data supporting the conclusions has been revealed in the tables (Table 1 and Table 4 in Appendix) of this manuscript.

\section{Authors' contributions}

LWY designed the study, performed the statistical analysis, and drafted the manuscript. LQ checked the data and statistical analysis, and wrote the manuscript. LWY and LQ contributed equally to this work as first authors. $\mathrm{HQH}$ and LYQ carried out the data collection and follow-up. XSH collected and organized the data. CSM and LAH conceived of the study, participated in its design and revised the manuscript. All authors have read and approved the final version of this manuscript for submission.

\section{Competing interests}

The authors declare that they have no competing interests.

\section{Consent for publication}

Not applicable

\section{Ethics approval and consent to participate}

This study was approved by the Institutional Research Ethics Committee of Sun Yat-sen University Cancer Center (YB2016-034). Because the research data was from cancer registry system, the privacy of any personal information has not been involved, and the need for providing consent to participate has been waived by Sun Yat-sen University Cancer Center IRB.

\section{Author details}

'Department of Cancer Prevention Research, Sun Yat-sen University Cancer Center, State Key Laboratory of Oncology in South China; Collaborative Innovation Center Medicine, 21 Qingcaigang, Jianshe 6 road, Guangzhou, Guangdong, China. ${ }^{2}$ School of Public Health, Sun Yat-sen University, 74 Zhongshan 2 road, Guangzhou, China. ${ }^{3}$ Sihui Cancer Institute, Sihui, Guangdong, China.

Received: 18 March 2016 Accepted: 26 September 2016 Published online: 12 October 2016

\section{References}

1. Ferlay J, Soerjomataram I, Ervik M, Dikshit R, Eser S, Mathers C, Rebelo M, Parkin DM, Forman D, Bray F. GLOBOCAN 2012 v1.0, Cancer Incidence and Mortality Worldwide: IARC CancerBase No. 11 [Internet]. Lyon: International Agency for Research on Cancer; 2013. http://globocan.iarc.fr. Accessed 20 Nov 2015.

2. El-Serag HB. Hepatocellular Carcinoma. New Engl J Med. 2011;365:1118-27.

3. Bosetti C, Turati F, La Vecchia C. Hepatocellular carcinoma epidemiology. Best Pract Res Clin Gastroenterol. 2014:28(5):753-70.

4. Tang D, Nagano H, Nakamura M, Wada H, Marubashi S, Miyamoto A, et al. Clinical and pathological features of Allen's type $C$ classification of resected combined hepatocellular and cholangiocarcinoma: a comparative study with hepatocellular carcinoma and cholangiocellular carcinoma. J Gastrointest Surg. 2006;10(7):987-98.

5. El-Serag HB. Epidemiology of Viral Hepatitis and Hepatocellular Carcinoma Gastroenterology. 2012;142(6):1264-73.

6. Franceschi S, Raza SA. Epidemiology and prevention of hepatocellular carcinoma. Cancer Lett. 2009;286:5-8.

7. Fan J, Wang J, Jiang Y, Xiang W, Liang H, Wei W, Qiao Y, Boffetta P. Attributable Causes of Liver Cancer Mortality and Incidence in China. Asian Pac J Cancer Prev. 2013;14(12):7251-6.
8. Sithithaworn P, Yongvanit P, Duenngai K, Kiatsopit N, Pairojkul C. Roles of liver fluke infection as risk factor for cholangiocarcinoma. J Hepatobiliary Pancreat Sci. 2014;21(5):301-8.

9. Qian MB, Utzinger J, Keiser J, et al. Clonorchiasis. Lancet. 2016;387:800-10.

10. Chuang S, Vecchia CL, Boffetta P. Liver cancer: Descriptive epidemiology and risk factors other than HBV and HCV infection. Cancer Lett. 2009:286(1):9-14.

11. Shih WL, Chang HC, Liaw YF, Lin SM, Lee SD, Chen PJ, et al. Influences of tobacco and alcohol use on hepatocellular carcinoma survival. Int J Cancer. 2012;131(11):2612-21.

12. Chen W, Zheng R, Zhang S, Zhao P, Zeng H, Zou X. Report of cancer incidence and mortality in China, 2010. Ann Transl Med. 2014;2(7):61.

13. Chen WQ, Zou XN, Zhang SW. Analysis of liver cancer geographical characteristics in China. Shi Yong Zhong Liu Xue Za Zhi. 2008;22(3):201-3 [in Chinese].

14. Gao S, Yang W, Bray F, Va P, Zhang W, Gao J, Xiang Y. Declining rates of hepatocellular carcinoma in urban Shanghai: incidence trends in 1976-2005. Eur J Epidemiol. 2012:27(1):39-46.

15. Chen JG, Zhu J, Zhang YH, et al. Incidence trend of liver cancer: an analysis of 40 year's data from Qidong population-based cancer registry. Zhong Guo Zhong Liu. 2014;23(8):621-8[in Chinese].

16. Wei ZL, Liang RX, Wang KB, et al. An Analysis of Trend on Incidence with Liver Cancer in Fusui, Guangxi 1974 2003. Zhong Guo Zhong Liu. 2007; 16(9):679-80 [in Chinese].

17 Li YH, Huang QQ. A Brief Inrtoduction on Cancer Registration in Sihui City. Zhong Guo Zhong Liu. 2003;12(9):21-2 [in Chinese].

18 Huang QH, Ling QN, Li YH, et al. An Analysis of Cancer Incidence in Sihui City. Zhong Guo Zhong Liu. 2005;14(4):241-3 [in Chinese].

19 Li YH, Huang QH, Lin X, et al. Survival rates of malignancies and nasopharyngeal carcinoma during 2003-2005 in Sihui city. Zhong Hua Zhong Liu Fang Zhi Za Zhi. 2013:20(10):734-44 [in Chinese].

20 Curado MP, Edwards B, Shin HR, Storm H, Ferlay J, Heanue M, et al. Cancer incidence in five continents volume IX. Lyon: IARC Sci Publ. 2008;160:99-100.

$21 \mathrm{Kim} \mathrm{H-j}$, Fay MP, et al. Permutation Tests for joinpoint regression with Applications to Cancer Rates. Stat Med. 2000;19:335-51.

22 Cayuela A, Rodríguez-Domínguez S, López-Campos JL, Vigil E. Age-periodcohort analysis of lung cancer mortality rates in Andalusia, 1975-2004. Lung Cancer. 2007:57(3):261-5.

23 Yuri Ito AITN. Comparison of Trends in Cancer Incidence and Mortality in Osaka, Japan, Using an Age-Period-Cohort Model. Asian Pac J Cancer Prev. 2011;12:879-88

24 Clayton D, Schifflers E. Models for temporal variation in cancer rates. I:AgePeriod and Age-Cohort Models. Stat Med.1987;6(4):449-67.

25 Clayton D, Schifflers E. Models for temporal variation in cancer rates. II: Ageperiod-cohort models. Stat Med. 1987;6(4):469-81.

26 Rosenberg PS, Anderson WF. Age-Period-Cohort Models in Cancer Surveillance Research: Ready for Prime Time? Cancer Epidem Biomar. 2011; 20(7):1263-8.

27 Du J, Lin X, Zhang L, Li Y, Xie S, Yang M, Guo J, Lin E, Liu Q, Hong M, et al. Secular trend analysis of lung cancer incidence in Sihui city, China between 1987 and 2011. Chin J Cancer. 2015;34:33.

28 Wu CG, Han K, Shao XP, Shu J, et al. Sero-epidemiological Study on Hepatitis B Virus Infection in Guangdong Province. Zhong Guo Yu Fang Yi Xue Za Zhi. 2009:10(5):350-4 [in Chinese].

29 Dai ZC, Qi GM. Viral hepatitis seroepidemiological survey in Chinese population, 1992-1995 (part two). Beijing: China Science and Technical Documents Publishing House; 1997. p. 105-14 [in Chinese].

30 Xiao JP, Zhang JK, Wu CG, et al. Impact of hepatitis B vaccination among children in Guangdong Province, China. Int J Infect Dis. 2012;16(9):692-6.

31 Chen D. Hepatitis B, vaccination: The key towards elimination and eradication of hepatitis B. J Hepatol. 2009;50(4):805-16.

32 Sun Z, Ming L, Zhu X, Lu J. Prevention and control of hepatitis B in China. J Med Virol. 2002;67(3):447-50

33 Fang YY, Wu J, Liu Q, et al. Investigation and analysis on epidemic status of clonorchiasis in Guangdong Province, China. J Pathog Biol. 2007;2(1):54-6 [in Chinese]

34 The Fourth National People's Congress. The Twelfth Five-Year Plan outline of national economic and social development. Beijing: People's Publishing House; 2011. p. 3-6.

35 Sherlock S. Viruses and hepatocellular carcinoma. Gut. 1994;35:828-32.

36 Kiyosawa K, Sodeyama T, Tanaka E, Gibo Y, Yoshizawa K, Nakano Y, Furuta S, Akahane $Y$, Nishioka K, Purcell RH. Interrelationship of blood transfusion, 
non-A, non-B hepatitis and hepatocellular carcinoma: analysis by detection of antibody to hepatitis C virus. Hepatology. 1990;12:671-5.

37 Yen FS, Shen KN. Epidemiology and early diagnosis of primary liver cancer in China. Adv Cancer Res. 1986:47:297-329.

38 Yang BH, Tang ZQ. Evaluation of Real-time Ultrasonography Used in Mass Screening of Primary Liver Cancer. Zhong liu. 1989;9(8):118-9 [In Chinese].

39 Samelson EJ, YZDP. Effect of Birth Cohort on Risk of Hip Fracture: AgeSpecific Incidence Rates in the Framingham Study. Am J Public Health. 2002;92(5):858-62

40 Han J, Chen XG. A Meta-Analysis of Cigarette Smoking Prevalence among Adolescents in China: 1981-2010. Int J Environ Res Public Health. 2015:12:4617-30.

41 Cheng XM, Fang JQ. Alcohol Consumption and Blood Pressure: A Nation-wide Survey of in 1991 in China. Gao Xue Ya Za Zhi. 1995;3(Suppl):50-4 [In Chinese].

Submit your next manuscript to BioMed Central and we will help you at every step:

- We accept pre-submission inquiries

- Our selector tool helps you to find the most relevant journal

- We provide round the clock customer support

- Convenient online submission

- Thorough peer review

- Inclusion in PubMed and all major indexing services

- Maximum visibility for your research

Submit your manuscript at www.biomedcentral.com/submit
Biomed Central 